\title{
Avaliação de toxicidade de biopesticida comercial à base de Bacillus thuringiensis em tilapia-do-Nilo, Oreochromis niloticus
}

Toxicity assessment of a comercial Bacillus thuringiensis-based insecticide on Nile tilápia, Oreochromis niloticus

Evaluación de la toxicidade de un insecticida comercial a base de Bacillus thuringiensis em la tilápia del Nilo, Oreochromis niloticus

Recebido: 03/06/2021 | Revisado: 13/06/2021 | Aceito: 21/06/2021 | Publicado: 04/07/2021

\author{
Alexia Marta Turchetto Simão \\ ORCID: https://orcid.org/0000-0002-1218-4340 \\ Universidade Federal do Tocantins, Brasil \\ E-mail: alexiatur@uft.edu.br \\ Ducilene do Carmo da Silva \\ ORCID: https://orcid.org/0000-0001-8074-7486 \\ Universidade Federal do Tocantins, Brasil \\ E-mail: ducycarmo045@gmail.com \\ Marco Aurélio Miranda Soares \\ ORCID: https://orcid.org/0000-0002-4473-2161 \\ Universidade Federal do Tocantins, Brasil \\ E-mail: marcoms0@ hotmail.com \\ Geraldo Vinicios Barbosa Dias \\ ORCID: https://orcid.org/0000-0002-3612-4035 \\ Universidade Federal do Tocantins, Brasil \\ E-mail: vinicios_cortez@hotmail.com \\ Ericsson Rubens Rodrigues Ferreira \\ ORCID: https://orcid.org/0000-0003-4566-4583 \\ Universidade Federal do Tocantins, Brasil \\ E-mail: ericsson@uft.edu.br \\ Sandro Estevan Moron \\ ORCID: https://orcid.org/0000-0002-1359-6160 \\ Universidade Federal do Tocantins, Brasil \\ E-mail: sandromoron@uft.edu.br \\ Wagner dos Santos Mariano \\ ORCID: https://orcid.org/0000-0003-0225-6889 \\ Universidade Federal do Tocantins, Brasil \\ E-mail:wagnermariano@uft.edu.br \\ Marcelo Gustavo Paulino \\ ORCID: https://orcid.org/ 0000-0003-0464-6848 \\ Universidade Federal do Tocantins, Brasil \\ E-mail: marcelopaulino@uft.edu.br
}

\begin{abstract}
Resumo
Os biopesticidas são usados como uma alternativa ao uso de pesticidas químicos sintéticos. As formulações que contêm esporos de Bacillus thuringiensis (Bt) são amplamente utilizados, entretanto, pouco se sabe dos efeitos na biota aquática. O objetivo do estudo foi avaliar os efeitos genotóxicos e histopatológicos de uma concentração subletal de um biopesticida comercial à base de Bt em Oreochromis niloticus. Os peixes foram divididos $(\mathrm{n}=10)$ em um grupo controle (GC) e um grupo exposto (GBt) na concentração de $60 \mathrm{mg} \mathrm{L}^{-1}$ do biopesticida Dipel® WP por 48 horas. Após a exposição, amostras de sangue, brânquias, fígado e rim foram coletadas para análise de biomarcadores de toxicidade. Os resultados demonstram que uma exposição aguda ao Bt induz o aumento de núcleos eritrocitários com morfologia segmentada. Não foi observado a indução de micronúcleos. As análises histopatológicas demonstraram que em 48 horas de exposição, no GBt, houve uma diminuição na frequência de hepatócitos hipertrofiados. Entretanto, o biopesticida não comprometeu a morfologia dos tecidos em toxicidade aguda. Os pesticidas de modo geral, são aplicados constantemente e regulamentações de uso não são amplamente seguidas, assim, nossos resultados sugerem que concentrações subletais podem induzir efeitos quando expostos de modo agudo. Os biopesticidas à base de Bt em baixas concentrações parecem ser pouco tóxicos aos peixes.
\end{abstract}

Palavras-chave: Biomarcadores; Citotoxicidade; Alteração nuclear; Histopatologia; Teleósteo. 


\begin{abstract}
Biopesticides are used as an alternative to synthetic chemical pesticides application. Formulations based on Bacillus thuringiensis $(\mathrm{Bt})$ spores are widely used, however, little is known about their effects on aquatic biota. The aim of the study was to evaluate the genotoxic and histopathological effects of a sublethal concentration of a commercial Btbased biopesticide on Oreochromis niloticus. The fish were divided $(\mathrm{n}=10)$ into a control group $(\mathrm{CG})$ and an exposed group (GBt) at a concentration of $60 \mathrm{mg} \mathrm{L}^{-1}$ of the biopesticide Dipel® WP for 48 hours. After exposure, blood, gill, liver and kidney samples were collected for analysis of toxicity biomarkers. The results demonstrate that an acute exposure to $\mathrm{Bt}$ induces the increase of erythrocyte nuclei with segmented morphology. No micronucleus induction was observed. Histopathological analysis showed that within 48 hours of exposure, in GBt, there was a decrease in the frequency of hypertrophied hepatocytes. However, the biopesticide did not compromise tissue morphology in acute toxicity. Pesticides in general are constantly applied and usage regulations are not widely followed, our results suggest that sublethal concentrations can induce effects when exposed acutely. Biopesticides based on Bt in low concentrations appear to be less toxic to fish.
\end{abstract}

Keywords: Biomarkers; Cytotoxicity; Nuclear alteration; Histopathology; Teleost.

\title{
Resumen
}

Los bioplaguicidas se utilizan como alternativa a la aplicación de pesticidas químicos sintéticos. Las formulaciones basadas en esporas de Bacillus thuringiensis (Bt) se utilizan ampliamente, sin embargo, se sabe poco acerca de sus efectos sobre la biota acuática. El objetivo del estudio fue evaluar los efectos genotóxicos e histopatológicos de una concentración subletal de un bioplaguicida comercial basado en Bt sobre Oreochromis niloticus. Los peces se dividieron $(\mathrm{n}=10)$ en un grupo de control (CG) y un grupo expuesto (GBt) a una concentración de $60 \mathrm{mg} \mathrm{L}^{-1} \mathrm{del}$ biopesticida Dipel® WP durante 48 horas. Después de la exposición, se recolectaron muestras de sangre, branquias, hígado y riñón para analizar los biomarcadores de toxicidad. Los resultados demuestran que una exposición aguda a Bt induce el aumento de núcleos de eritrocitos con morfología segmentada. No se observó inducción de micronúcleos. Los análisis histopatológicos mostraron que dentro de las 48 horas posteriores a la exposición, en GBt, hubo una disminución en la frecuencia de hepatocitos hipertrofiados. Sin embargo, el bioplaguicida no comprometió la morfología del tejido en la toxicidad aguda. Los pesticidas en general se aplican constantemente y las regulaciones de uso no se siguen ampliamente, por lo que nuestros resultados sugieren quelas concentraciones subletales pueden inducir efectos cuando se exponen de forma aguda. Los bioplaguicidas basados en Bt en concentraciones bajas parecen ser menos tóxicos para los peces.

Palabras clave: Biomarcadores; Citotoxicidad; Alteración nuclear; Histopatología, Teleósteos.

\section{Introdução}

Pesticidas químicos são amplamente utilizados para o controle de pragas que prejudicam a produção agrícola, no entanto, o uso indiscriminado representa sérios riscos ambientais (Silva \& Santos, 2007; Sharma \& Singhvi, 2017). Com baixa especificidade, esses químicos afetam uma grande quantidade de organismos, muitos considerados não-alvo (Meher et al., 2002; Chaudhary et al., 2017; Schweizer et al., 2019). Frente aos riscos associados ao uso de pesticidas sintéticos, há um crescente interesse em alternativas menos agressivas ao ambiente para o controle de pestes na agricultura (Pino-Otín et al., 2019). Neste cenário, os biopesticidas aparecem no mercado como uma alternativa menos prejudicial ao meio ambiente (Cruz et al., 2004; Oliveira-Filho, 2008; Artega et al., 2014), pois dispõe de maior especificidade com o organismo-alvo, afetando um número menor de organismos não-alvo (Glare et al., 2012; Villaverde et al., 2014).

Dentre os biopesticidas mais utilizados, estão aqueles à base da bactéria Bacillus thuringiensis (Bt) (Bravo et al., 2011). Os produtos à base de Bt são utilizados como inseticida principalmente contra larvas da ordem Lepidoptera (Freire et al., 2014; Venter \& Bohn, 2016). Durante a esporulação, o Bt produz inclusões cristalinas protéicas (proteínas Cry), as quais são ingeridas pelo inseto provocando aumento de permeabilidade intestinal e eventual ruptura celular, levando à morte por inanição (Kitada et al., 2006; Martins et al., 2008).

Embora considerados inertes a muitas espécies, não se pode desconsiderar que biopesticidas formulados sejam completamente seguros (Copping \& Menn, 2000; Murussi et al., 2015). De acordo com Villaverde et al. (2014), alguns biopesticidas à base de Bt possuem um espectro relativamente amplo de organismos alvo. Ainda, alguns estudos apontam o Bt como agente nocivo a organismos não alvos como tartarugas (Chen et al., 2014) e peixes (Omoya \& Akharaiyi, 2015; Mariano et al., 2019). 
A avaliação dos efeitos ambientais em organismos aquáticos pode ser feita através de ferramentas que oferecem informações sensíveis do efeito de contaminantes sobre os organismos (Vieira et al., 2017). Tecidos como sangue, brânquias, fígado e rim dos peixes são amplamente utilizados como biomarcadores de toxicidade em peixes. As células do sangue são as primeiras a ter contato com qualquer contaminante e podem ser afetadas pelos xenobióticos, além disso, respondem a alterações que ocorram em outros tecidos com disfunções morfofisiológicas (Sadauskas-Henrique et al., 2011).

Anormalidades no núcleo de eritrócitos são, portanto, ótimos indicadores de potencial citotóxico, e as técnicas de análise bastante sensíveis aos efeitos de contaminantes na integridade celular (Azevedo et al., 2012). Associados a isso, análises histopatológicas em diferentes tecidos permitem uma localização intermediária em relação ao nível de organização biológica, uma vez que alterações podem aparecer como resposta a concentrações subletais à estressores (Bernet et al., 1999).

Em vista da ampla utilização dos biopesticidas à base de Bt, é necessário considerar que a utilização demasiada destes produtos pode aumentar a concentração disponível no ambiente (Oliveira-Filho, 2008) e afetar indiretamente os organismos não-alvos. Com base nisso e pela falta de informações que nos permite entender como ocorre o mecanismo de ação do Bt em vertebrados ou seu potencial de toxicidade que são poucos conhecidos. Dessa forma, o objetivo do estudo foi avaliar os efeitos genotóxicos e morfológicos de uma concentração subletal de um biopesticida comercial à base de Bt em Oreochromis niloticus após exposição aguda.

\section{Metodologia}

\subsection{Animais}

Os peixes provenientes da Piscicultura Araguatins, estado do Tocantins, foram transportados para o Laboratório de Morfofisiologia Animal Comparada da Universidade Federal do Tocantins (UFT), campus Araguaína. Os peixes foram aclimatados por 30 dias em tanques de $250 \mathrm{~L}$ com aeração constante, mantidos à temperatura de $23^{\circ} \mathrm{C} \pm 1^{\circ} \mathrm{C}$ e fotoperiodo natural (12:12h), e alimentados ad libitum com ração comercial 40\% proteína uma vez ao dia.

\subsection{Biopesticida}

O biopesticida empregado no experimento foi a formulação comercial do inseticida biológico Dipel@ WP, à base de Bacillus thuringiensis var. kurstaki, linhagem HD-1. A formulação contém $33,6 \mathrm{~g} \mathrm{~L}^{-1}$ de esporos ativos de Bt, correspondendo a 3,36\% da razão $\mathrm{m} / \mathrm{v}$ da formulação (16.000 UI de potência por mg; mínimo de 25 bilhões de esporos viáveis por grama de produto).

\subsection{Delineamento experimental e coleta de amostras}

Juvenis de $O$. niloticus $(47,28 \pm 3,0 \mathrm{~g} ; 14,59 \pm 0,36 \mathrm{~cm})$ foram aleatoriamente divididos em dois grupos $(\mathrm{n}=10) \mathrm{e}$ colocados em aquários de $100 \mathrm{~L}$. O grupo tratado (GBt) foi exposto à concentração de $60 \mathrm{mg} \mathrm{L}^{-1}$ do biopesticida por 48 horas. O grupo controle (GC) foi exposto sob as mesmas condições, porém, livre de contaminante. Após exposição, os peixes foram anestesiados com benzocaína $\left(0,1 \mathrm{~g} \mathrm{~L}^{-1}\right)$ e amostras de sangue $(0,5 \mathrm{~mL})$ foram obtidas via punção caudal, com auxílio de seringas heparinizadas, para avaliação de potencial genotóxico e mutagênico. Em seguida, os peixes foram mortos por secção medular e foram coletados brânquias, fígado e rim. Os órgãos foram fixados em solução Bouin para análises histopatológicas.

O procedimento experimental foi aprovado pelo Comitê de Ética de Uso de Animais - UFT (CEUA) sob o número 23101.001.738/2018-99. 


\subsection{Biomarcadores genotóxicos}

Uma alíquota de sangue foi utilizada para avaliar a viabilidade eritrocitária através do teste de exclusão de células inviáveis pelo corante azul de tripam. A viabilidade foi de $100 \%$ e assim, extensões sanguíneas foram fixadas em metanol e coradas com Giemsa 5\%.

Para o teste do micronúcleo (MN), foi utilizada a metodologia empregada por Hooftman \& Raat (1982) com modificações de Cavalcante et al. (2008). Para considerar um micronúcleo genuíno, seguiu-se um critério característico das análises: (1) ser morfologicamente semelhante ao núcleo principal; (2) ter um diâmetro de até 1/3 do diâmetro do núcleo principal; (3) não ser refringente; (4) não estar de qualquer forma conectado ao núcleo principal; (5) possuir a mesma coloração do núcleo principal.

A ocorrência de alterações nucleares eritrocitárias (ANE) foi segundo metodologia de Carrasco et al. (1990), modificada por Cavalcante et al. (2008). Para as análises das ANE foram considerados os núcleos segmentados, indentados, lobulados, binucleados e reniformes. Todas as análises foram feitas em 2000 células utilizando um microscópio optico (Leica DM500) sob magnificação 1000x.

\subsection{Biomarcadores Morfológicos}

As amostras fixadas de brânquias, fígado e rim foram incluídas em parafina, cortadas em $5 \mu \mathrm{m}$ e coradas com hematoxilina e eosina (H\&E). Análises histopatológicas foram realizadas pelo método de análise cega em microscópio de luz Leica DM500. Todas as análises histopatológicas foram quantificadas aleatoriamente em 5 secções x 5 campos por secção ( $\mathrm{n}=25$ campos aleatórios) por animal.

O comprometimento morfofuncional dos órgãos (Índice de lesão do órgão - $\mathrm{I}_{\mathrm{org}}$ ) foi determinado com base na metodologia de Bernet et al. (1999) com adaptações de Paulino et al. (2020). Brevemente foi atribuído um escore numérico (Sc) e um fator de importância (Fi) de cada lesão. O Sc dado ao intervalo de frequência com a qual uma patologia é identificada nos campos amostrais para cada animal, compreende: (0) lesão ausente; (1) raramente presente; (2) pouco frequente; (3) moderadamente frequente; (4) muito frequente. O Fi indica como uma lesão afetaria a função do órgão e a sobrevivência do peixe, onde: (1) são lesões facilmente reversíveis e com pouca importância patológica; (2) lesões reversíveis quando o agente estressor é neutralizado, portanto, de moderada importância patológica, e; (3) lesões geralmente irreversíveis e de extrema importância patológica.

O $\mathrm{I}_{\text {alt }}$ foi determinado pela multiplicação do escore pelo fator de importância $\left(\mathrm{I}_{\text {alt }}=\mathrm{Sc} \times \mathrm{Fi}\right)$, enquanto o $\mathrm{I}_{\mathrm{org}}$ foi determinado pela soma dos valores de $\mathrm{I}_{\text {alt }}$ de cada lesão para cada animal ( $\left.\mathrm{I}_{\text {org }}=\sum \mathrm{I}_{\text {alt }}\right)$.

\subsection{Análise estatística}

Os dados estão apresentados como média e erro padrão da média ( \pm EPM). Os grupos GC e GBt foram comparados entre si por meio do teste t de Student não-paramétrico (Mann-Whitney), com intervalo de confiança $\mathrm{p}<0.05$.

\section{Resultados}

\subsection{Biomarcadores genotóxicos e mutagênicos}

Não houve presença de micronúcleos após a exposição ao biopesticida, entretanto, as ANEs apresentam um aumento de 137\% de núcleos segmentados (Tabela 1). 
Tabela 1. Valores médios ( \pm E.P.M) da avaliação genotóxica e mutagênica em eritrócitos de Oreochromis niloticus expostos ao biopesticida à base de Bacillus thuringiensis.

\begin{tabular}{|c|c|c|}
\hline & \multicolumn{2}{|c|}{ Exposição ao Dipel® } \\
\hline & Controle & $60 \mathrm{mg} \mathrm{L}^{-1}$ \\
\hline Núcleo binucleado & 0 & 0 \\
\hline Núcleo indentado & $2,4 \pm 0,9$ & $4,1 \pm 1,0$ \\
\hline Núcleo lobulado & 0 & $0,1 \pm 0,1$ \\
\hline Núcleo reniforme & $7,0 \pm 1,9$ & $10,9 \pm 2,5$ \\
\hline Núcleo segmentado & $2,4 \pm 0,9$ & $5,7 \pm 1,1 *$ \\
\hline Núcleo vacuolado & 0 & 0 \\
\hline Micronúcleo & 0 & 0 \\
\hline
\end{tabular}

(*) indica diferenças estatísticas ( $p>0,05)$ em relação ao grupo controle. Fonte: Autores.

\subsection{Biomarcadores morfológicos}

O índice de alteração individual $\left(\mathrm{I}_{\mathrm{alt}}\right)$ para rim, fígado e brânquias está representado na Tabela 2. Não houve alteração no tecido renal ou em brânquias dos animais do grupo exposto ao Bt quando comparados ao grupo controle. No fígado, houve diminuição de hepatócitos hipertrofiados em animais expostos ao biopesticida (Figura 1). Não houve alterações dos tecidos que levariam a uma alteração severa de suas funções. O I org para cada tecido analisado está representado na Figura 2.

Tabela 2. Índice de alteração individual $\left(\mathrm{I}_{\mathrm{alt}}\right)$ de histopatologias encontradas em rim, fígado e brânquias de Oreochromis niloticus expostos ao biopesticida à base de Bacillus thuringiensis.

\begin{tabular}{|c|c|c|c|}
\hline & \multicolumn{3}{|c|}{ Exposição de 48 h } \\
\hline & $\mathrm{Fi}$ & Controle & $60 \mathrm{mg} \mathrm{L}^{-1}$ \\
\hline \multicolumn{4}{|l|}{ Patologias renais } \\
\hline Alterações nucleares do epitélio renal & 1 & 0 & $0,5 \pm 0,5$ \\
\hline Atrofia (túbulos e glomérulos) & 2 & $1,7 \pm 0,3$ & $0,5 \pm 0,5$ \\
\hline Hiperplasia dos glomérulos & 2 & $1,1 \pm 0,4$ & $1,5 \pm 0,5$ \\
\hline Hiperplasia dos túbulos & 2 & $1,7 \pm 0,3$ & $2,0 \pm 0,0$ \\
\hline Hipertrofia dos glomérulos & 1 & $0,9 \pm 0,1$ & $0,7 \pm 0,2$ \\
\hline \multicolumn{4}{|l|}{ Patologias hepáticas } \\
\hline Acúmulos de substâncias intracelulares & 1 & $4,0 \pm 0,0$ & $3,8 \pm 0,2$ \\
\hline Alterações nucleares & 1 & $0,2 \pm 0,1$ & 0 \\
\hline Atrofia celular & 2 & $0,4 \pm 0,3$ & 0 \\
\hline Degeneração citoplasmática & 1 & $0,3 \pm 0,2$ & $0,1 \pm 0,1$ \\
\hline Hipertrofia dos hepatócitos & 1 & $0,6 \pm 0,3$ & $0,1 \pm 0,1 *$ \\
\hline \multicolumn{4}{|l|}{ Patologias branquiais } \\
\hline Aneurisma & 1 & $0,1 \pm 0,1$ & 0 \\
\hline Alterações circulatórias & 1 & 0 & $0,2 \pm 0,1$ \\
\hline Células pilares & 1 & $1,4 \pm 0,3$ & $1,2 \pm 0,2$ \\
\hline Descolamento do epitélio lamelar e edemas & 1 & $0,6 \pm 0,3$ & $0,7 \pm 0,3$ \\
\hline Hiperplasia do epitélio & 2 & $2,0 \pm 0,5$ & $2,9 \pm 0,7$ \\
\hline Hipertrofia do epitélio lamelar & 1 & $3,7 \pm 0,2$ & $3,3 \pm 0,4$ \\
\hline Ruptura do epitélio & 2 & $0,2 \pm 0,2$ & $0,2 \pm 0,2$ \\
\hline
\end{tabular}

(*) indica diferença estatística em relação ao grupo controle $(\mathrm{p}<0,05)$.

$\mathrm{Fi}=$ Fator de Impacto. Fonte: Autores. 
Figura 1. Morfologia e histopatologias de rim, fígado e brânquias de Oreochromis niloticus após exposição aguda (48 h) ao biopesticida à base de Bacillus thuringiensis. (A) Tecido renal do grupo controle. Seta contínua indica o glomérulo e seta tracejada mostrando túbulos renais. (B e C) Alterações observadas em animais expostos ao Bt. Cabeça de seta indica atrofia dos glomérulos e seta dupla indica hipertrofia dos glomérulos. (D) Arquitetura hepática normal do grupo controle. Seta contínua demonstram os hepatócitos morfologicamente normais. Setas tracejadas demonstram os capilares sinusoides. (E e F) Tecido hepático do grupo exposto. Asteriscos indicam os hepatócitos hipertrofiados. Cabeça de seta mostrando acúmulo de substâncias intracelulares e cabeça de seta dupla indicando degeneração citoplasmática. (G) Tecido branquial normal do grupo controle. Seta contínua indicando o epitélio lamelar íntegro. Seta tracejada demonstrando células pilares. (H e I) Alterações em brânquias de animais expostos. Cabeça de seta indica hipertrofia do epitélio lamelar. Cabeça de seta dupla demonstra hiperplasia do epitélio lamelar e asterisco indicando constrição da célula pilar. $\mathrm{F}=$ filamento branquial; $\mathrm{L}=$ lamela secundária; $\mathrm{CC}=$ célula cloreto $\mathrm{CM}=$ célula mucosa. Coloração: Hematoxilina \& Eosina. Barra $=20 \mu \mathrm{m}$.
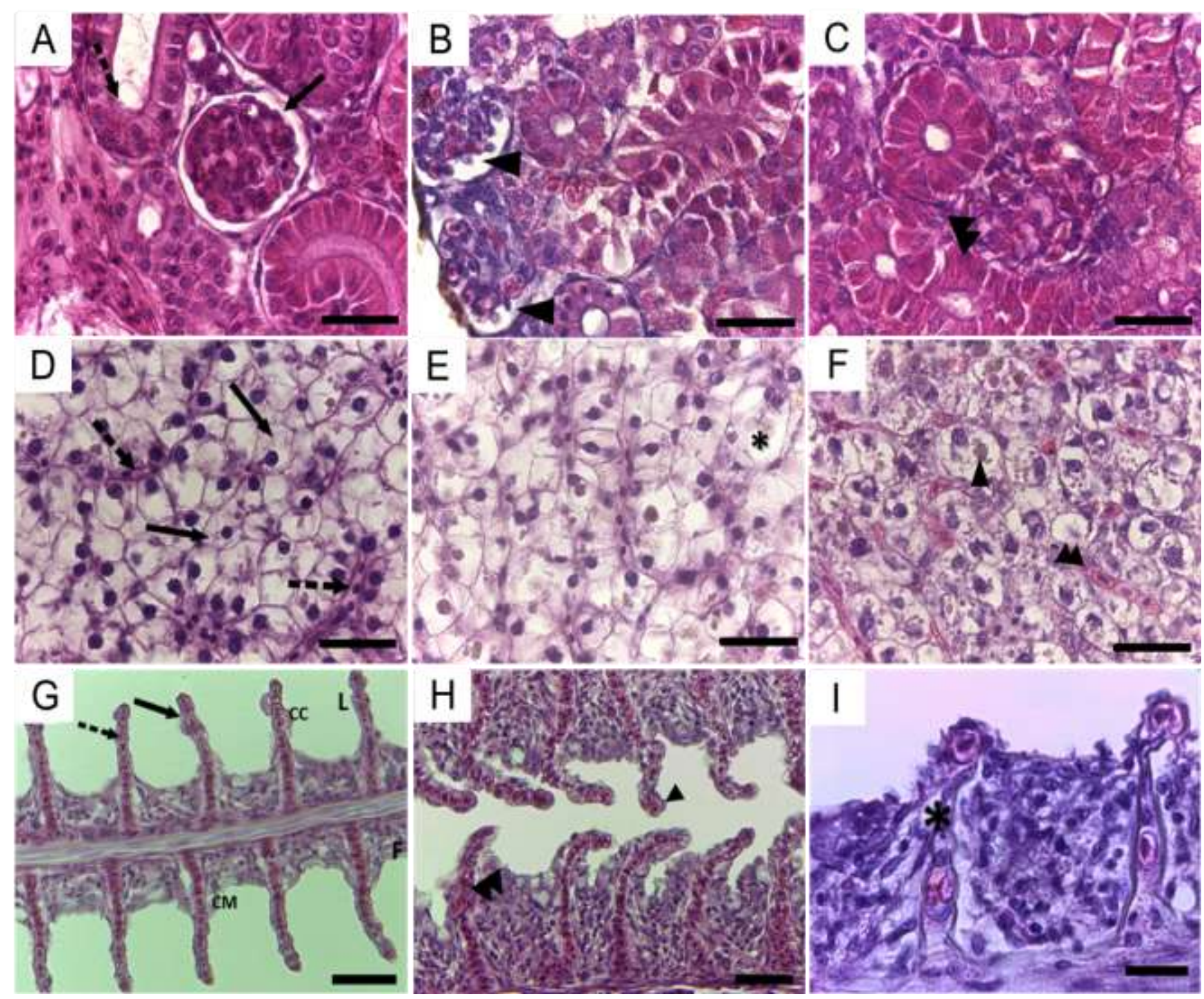

Fonte: Autores. 
Figura 2. Índice de alteração do órgão ( $\mathrm{I}_{\text {org }}$ em rim (A), fígado (B) e brânquia (C) de Oreochromis niloticus expostos ao biopesticida à base de Bacillus thuringiensis. $\mathrm{GC}=$ grupo controle; $\mathrm{GBt}=$ grupo exposto a $60 \mathrm{mg} \mathrm{L} \mathrm{L}^{-1}$ do herbicida.
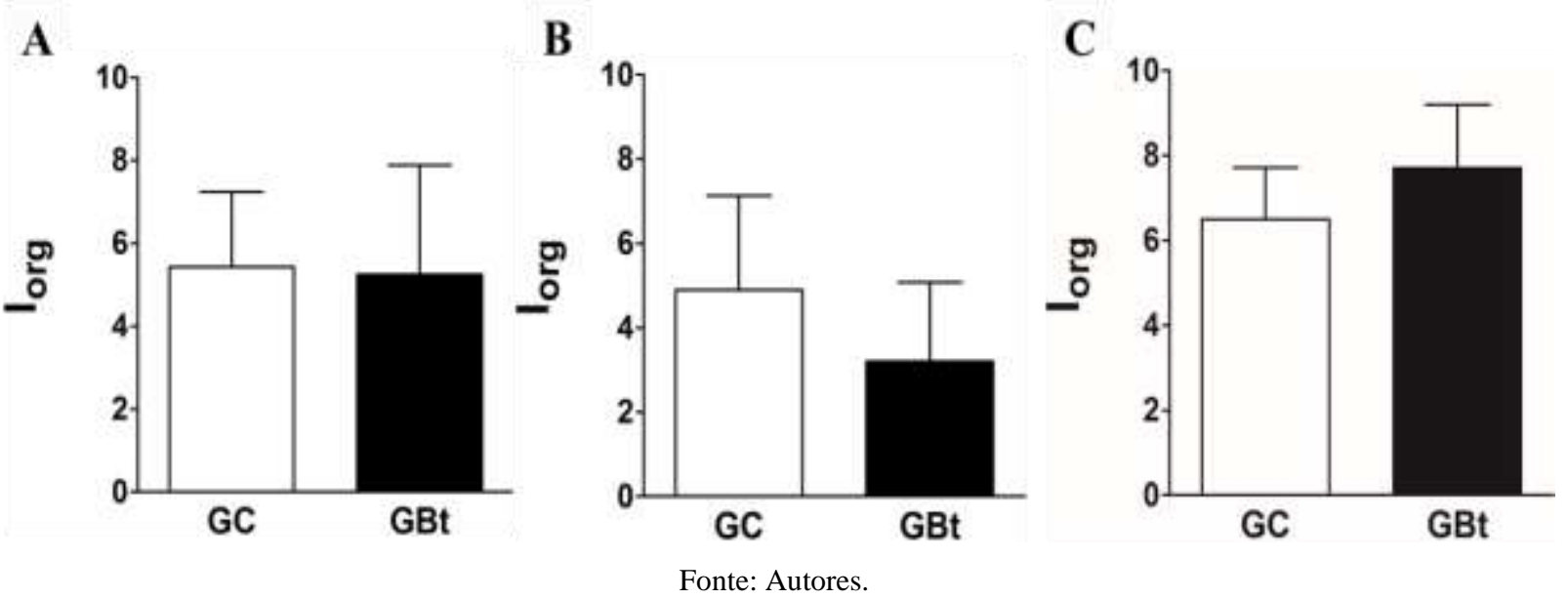

\section{Discussão}

O controle de insetos na agricultura com produtos químicos é um grande problema de poluição ambiental e efeitos danosos para a saúde humana. Adicionados a isso, a população de insetos tem se tornado resistentes a estes contaminantes, o qual tem causado um surto significativo de pragas secundárias (Meher et al., 2002; Devine \& Furlong, 2007). O uso de compostos contidos em biopesticidas é considerado seguro, entretanto, como apontam Mossa et al. (2018), a segurança e a seletividade desta categoria de pesticida não são totais.

A concentração utilizada neste estudo não ocasionou toxicidade em $O$. niloticus, embora, alguns estudos demonstram que este agente biológico pode alterar funções biológicas importantes dos vertebrados (Shaban et al., 2003; Chen et al., 2014; Mariano et al., 2019). No entanto, é necessário considerar a influência da forma de exposição do organismo ao agente, seja às protoxinas, às toxinas ativas, à bactéria ou aos esporos. Grisolia et al. (2009) destacaram a relevância da forma de exposição na toxicidade após testarem diferentes modos de exposição em peixes e ratos a três estirpes de Bacillus spp.

Douville et al. (2005) reportaram que algumas formulações de Bt podem ser mutagênicas para algumas espécies de organismos não-alvo como gafanhotos. Grisolia et al. (2009) inocularam em O. niloticus, por via intra-abdominal, $0.2 \mathrm{~mL}$ de três estirpes de Bacillus (Bt kurstaki, Bt israelensis e B. sphaericus H5). Após 72h, os autores identificaram aumento de necrose nos eritrócitos expostos a $B t$ israelensis e $B t$ kurstaki, porém não identificaram a ocorrência de apoptose, concluindo que das estirpes estudadas, nenhuma apresentou genotoxicidade. Neste estudo, não observamos citotoxicidade em eritrócitos e presença de micronúcleos a qual poderia estar atribuída a diversos fatores, como baixa concentração do biopesticida na água biodisponível à espécie que cause qualquer efeito fisiopatológico e genotóxico, ou relacionados ao ciclo celular. Fatores genotóxicos e mutagênicos que alteram o ciclo celular podem demonstrar efeitos após o término da divisão celular durante estágio de telófase e citocinese, desta forma, alguns autores relatam que micronúcleos em eritrócitos de peixes foram visíveis após exposição à xenobióticos (Udroiu, 2006; Disner et al, 2011; Del-Guercio et al, 2017).

Da mesma forma, Freire et al. (2014) não identificaram citotoxicidade e genotoxicidade em células sanguíneas de $O$. niloticus expostos aos cristais Cry1Ia, Cry10Aa e Cry1Ba6, produzidos por esporos Bt, após exposição aguda na água por $96 \mathrm{~h}$. Em insetos alvos, para ocorrer a ativação da toxina com efeito inseticida, as protoxinas das estirpes de Bt necessitam de $\mathrm{pH}$ alcalino para a solubilização e proteases específicas para a clivagem, uma condição encontrada no intestino dos insetos suscetíveis ao patógeno. Por isso, o uso do Bt é considerado seguro para a maioria dos organismos não-alvo (Oliveira et al., 2007). 
No entanto, neste estudo, núcleos segmentados foram evidentes após a exposição. A presença de ANEs é indicativo de danos citológicos e genéticos em peixes frente a diversos contaminantes (Ventura et al., 2008) e são descritas como danos análogos aos micronúcleos, uma vez que suas frequências são maiores após as exposições a agentes tóxicos (Ayllón \& GarciaVazquez, 2000). Não existe uma relação específica da ação do Bt com as ANEs, entretanto, a alteração em núcleos segmentados observados para $O$. niloticus poderiam estar relacionados a ajustes intracelulares na produção e manutenção da lâmina nuclear ocasionados pelo biopesticida.

Alterações histopatológicas podem resultar da exposição de xenobióticos e são respostas pouco específicas ao agente estressor que refletem a mudanças da morfologia dos tecidos a estímulos adversos (Wolf et al., 2015). As brânquias são bons indicadores de toxicidade pois é uma das principais vias de entrada de contaminantes em peixes, enquanto o rim e o fígado são excelentes indicadores que podem evidenciar efeitos metabólicos e de ação tóxica (Lins et al., 2010). Moraes et al. (2018) observaram alterações branquiais em Brycon amazonicus após exposição a 7,2 $\mu \mathrm{g} \mathrm{L} \mathrm{L}^{-1}$ a cipermetrina, enquanto Benli \& Özkul (2010), encontraram deformidades nas lamelas, hiperemia, proliferação de células do epitélio e cloreto, fusão de lamelas secundárias e telangiectasias em $O$. niloticus expostos a $0,5 \mathrm{mg} \mathrm{L}^{-1}$ a fenitrotion por $96 \mathrm{~h}$. Entretanto, após exposição ao biopesticida, em brânquias de $O$. niloticus não houve diferença na ocorrência de alterações entre o grupo mantido livre de contaminante e o grupo exposto ao Bt.

É comum, em exposições a agentes químicos e em exposições prolongadas, aparecer alterações dos túbulos e glomérulos de teleósteos (Pacheco \& Santos, 2002; Santos et al., 2012; Ranzani-Paiva et al., 2014). No presente trabalho, não houve alteração renal após a exposição aguda ao biopesticida. Isto sugere que, nas condições experimentais, o biopesticida à base de $B t$ não apresentou efeito nefrotóxico. Embora, os efeitos de xenobióticos e a severidade dos danos nos rins de peixes parece depender da sensibilidade das espécies às substâncias presentes no ambiente (Silva \& Martinez, 2007).

Alterações morfológicas no fígado podem representar estratégias adaptativas para manter as funções fisiológicas (Fanta et al., 2003; Liebel et al., 2013). De acordo com Wolf \& Wolfe (2005), o aumento do volume celular pode ser resultado de proliferação de organelas, falhas na divisão celular, degeneração e acúmulo de substâncias, além disto, a produção de vitelogenina pode levar à hipertrofia fisiológica. Após a exposição, notamos a redução na frequência de hepatócitos hipertrofiados em GBt. A partir das observações do parênquima hepático, as células aparentam conter acúmulo de glicogênio como mostrado por Wolf \& Wolfe (2005). Camargo \& Martinez (2007) associam a depleção das reservas de glicogênio à resposta de estresse ambiental. No presente estudo, a turbidez da água provocada pela adição do biopesticida poderia ser um estressor, no entanto, para determinação desta relação, seriam necessárias análises bioquímicas de glicose e cortisol plasmáticos.

Em exposição de ratos e coelhos a Bt, Meher et al. (2002) não identificaram alterações significativas em biomarcadores plasmáticos de dano hepático, porém, Shaban et al. (2003) observaram a indução de peroxidação lipídica e estresse oxidativo em fígado de ratos, atribuída à formulação de Dipel.

\section{Conclusão}

A concentração do biopesticida em exposição aguda delineada neste estudo indicou não toxicidade à tilápia-do-Nilo. Concentrações de xenobióticos em compartimentos ambientais que têm relevância ecológica são consideradas aquelas que podem ser encontradas no ambiente, após a emissão, dispersão e deposição final. Contudo, o aumento da frequência de núcleos segmentados após a exposição à formulação comercial do biopesticida pode indicar um efeito citotóxico e genotóxico. As alterações identificadas no fígado sugerem que, a nível metabólico, $O$. niloticus responde ao biopesticida utilizado no estudo. No entanto, são necessários estudos mais detalhados que integrem análises bioquímicas e histológicas para investigar a resposta de $O$. niloticus à exposição ao Bt em formulação comercial. 


\section{Referências}

Artega, M. E., Mancebo, A., Molier, T., Gómez, D., González, C., Bada, A. M., González, B., Rojas, N. M., \& Rodríguez, G. (2014). Dermal toxicity, eye and dermal irritation and skin sensitization evaluation of a new formulation of Bacillus thuringiensis var israelensis SH-14. Regulatory Toxicology and Pharmacology, 68, 147 - 151. 10.1016/j.yrtph.2013.12.003

Ayllón, F., \& Garcia-Vazquez, E. (2000). Induction of micronuclei and other nuclear abnormalities in European minnow Phoxinus phoxinus and mollie Poecilia latipinna: An assessment of the fish micronucleus test. Mutation Research, 467, 177 - 186. 10.1016/s1383-5718(00)00033-4

Azevedo, J. S., Braga, E. S., \& Ribeiro, C. A. O. (2012). Nuclear abnormalities in erythrocytes and morphometric indexes in the catfish Cathorops spixii (Ariidae) from different sites on the southeastern Brazilian coast. Brazilian Journal of Oceanography, 60(3), 323 - 330. https://www.scielo.br/j/bjoce/a/585HvcPvQhXCCrjM8hPPzpc/?lang=en

Benli, A. Ç. K., \& Özkul, A. (2010). Acute toxicity and histopathological effects of sublethal fenitrothion on Nile tilapia, Oreochromis niloticus. Pesticide Biochemistry and Physiology, 97, 32 - 35. 10.1016/j.pestbp.2009.12.001

Bernet, D., Schmidt, H., Meier, W., Burkhardt-Holm, P., \& Wahli, R. (1999). Histopathology in fish: proposal for a protocol to assess aquatic pollution. Journal of Fish Diseases, 22, 25 - 34. 10.1046/j.1365-2761.1999.00134.x

Bravo, A., Likitvivatanavong, S., Gill, S. S., \& Soberón, M. (2011). Bacillus thuringiensis: A story of a successful bioinsecticide. Insect Biochemistry and Molecular Biology, 41(7), 423 - 431. 10.1016/j.ibmb.2011.02.006

Camargo, M. M. P., \& Martinez, C. B. R. (2007). Histopathology of gills, kidney and liver of a Neotropical fish caged in an urban stream. Neotropical Ichthyology, 5(3), 327 - 336. 10.1590/S1679-62252007000300013

Carrasco, K. R., Tilbury, K. L., \& Myers, M. S. (1990). Assessment of the piscine micronucleus test as an in situ biological indicator of chemical contaminant effects. Canadian Journal of Fisheries and Aquatic Sciences, 47(11), 2123 - 2136. 10.1139/f90-237

Cavalcante, D., Martinez, C., \& Sofia, S. (2008). Genotoxic effects of Roundup ${ }^{\circ}$ on the fish Prochilodus lineatus. Mutation Research/Genetic Toxicology and Environmental Mutagenesis, 655, 41 - 46. 10.1016/j.mrgentox.2008.06.010

Chaudhary, S., Kanwar, R. K., Sehgal, A., Cahill, D. M., Barrow, C. J., Sehgal, R., \& Kanwar, J. R. (2017). Progress on Azadirachta indica based biopesticides in replacing synthetic toxic pesticides. Frontiers in Plant Science, 8, 1 - 13. 10.3389/fpls.2017.00610

Chen, J., Zhu, N., Kong, L., Bei, Y., Zheng, T., Ding, X., \& He, Z. (2014). First reported fatal Bacillus thuringiensis infections in Chinese soft-shelled turtles (Trionyx sinensis). Aquaculture, 428-429, 16 - 20. DOI: 10.1159/000479795

Copping, L. G., \& Menn, J. J. (2000). Biopesticides: a review of their action, applications and efficacy. Pest Management Science, 56, 651 - 676. 10.1002/1526-4998(200008)56:8<651::AID-PS201>3.0.CO;2-U

Cruz, C., Machado-Neto, J. G., \& Menezes, M. L. D. (2004). Toxicidade aguda do inseticida parationmetílico e do biopesticida azadiractina de folhas de neem (Azadirachta indica) para alevino e juvenil de pacu (Piaractus mesopotamicus). Pesticidas: Revista de Ecotoxicologia e Meio Ambiente, 14,93 - 102. 10.5380/pes.v14i0.3127

Del-Guercio, A. M. F., Christofoletti, C. A., \& Fontanetti, C. S. (2017). Evaluation of the domestic wastewater treatment efficiency by micronucleus test on Oreochromis niloticus (Cichlidae). Eng Sanit Ambient, 22(6), 1121-1128. 10.1590/S1413-4152201773709

Devine, G. J., \& Furlong, M. J. (2007). Insecticide use: Contexts and ecological consequences. Agriculture and Human Values, 24 , 281 - 306. $10.1007 / \mathrm{s} 10460-007-9067-\mathrm{Z}$

Disner, G. R., Rocha, M. V., \& Miranda, G. B. (2011). Avaliação da atividade mutagênica do Roundup® em Astyanax altiparanae (Chordata, Actinopterygii). Evidência, Joaçaba. 11(1) 33-42. https://portalperiodicos.unoesc.edu.br/evidencia/article/view/1384

Douville, M., Gagné, F., Masson, L., McKay, J., \& Blaise, C. (2005). Tracking the source of Bacillus thuringiensis Cry1Ab endotoxin in the environment. Biochemical Systematics and Ecology, 33, 219 - 232. 10.1016/j.bse.2004.08.001

Fanta, E., Rios, F. S., Romão, S., Vianna, A. C. C., \& Freiberger, S. (2003). Histopathology of the fish Corydoras paleatus contaminated with sublethal levels of organophosphorus in water and food. Ecotoxicology and Environmental Safety, 54(2), 119 - 130. 10.1016/s0147-6513(02)00044-1

Freire, I. S., Miranda-Vilela, A. L., Fascineli, M. L., Oliveira-Filho, E. C., Martins, E. S., Monnerat, R. G., \& Grisolia, C. K. (2014). Genotoxic evaluation in Oreochromis niloticus (Fish: Characidae) of recombinant spore-crystal complexes Cry1Ia, Cry10Aa and Cry1Ba6 from Bacillus thuringiensis. Ecotoxicology, 23, 267 - 272. 10.1007/s10646-013-1170-x

Glare, T., Caradus, J., Gelernter, W., Jackson, T., Keyhani, N., Köhl, J., Marrone, P., Morin, L., \& Stewart, A. (2012). Have biopesticides come of age? Trends in Biotechnology, 30(5), 250 - 258. 10.1016/j.tibtech.2012.01.003

Grisolia, C. K., Oliveira-Filho, E. C., Ramos, F. R., Lopes, M. C., Muniz, D. H. F., \& Monnerat, R. G. (2009). Acute toxicity and cytotoxicity of Bacillus thuringiensis and Bacillus sphaericus strains on fish and mouse bone marrow. Ecotoxicology, 18, 22 - 26. DOI 10.1007/s10646-008-0252-7

Hooftman, R. N., \& de Raat, W. K. (1982). Induction of nuclear anomalies (micronuclei) in the peripheral blood erythrocytes of the eastern mudminnow Umbra pygmaea by ethyl methanesulphonate. Mutation Research Letters, 104(1-3), 147 - 152. 10.1016/0165-7992(82)90136-1

Kitada, S., Abe, Y., Shimada, H., Kusaka, Y., Matsuo, Y., Katayama, H., Okumura, S., Akao, T., Mizuki, E., Kuge, O., Sasaguri, Y., Ohba, M., \& Ito, A. (2006). Cytocidal actions of parasporin-2, an anti-tumor crystal toxin from Bacillus thuringiensis. The Journal of Biological Chemistry, 281(36), 26350 26350. 10.1074/jbc.M602589200 
Liebel, S., Tomotake, M. E. M., \& Ribeiro, C. A. O. (2013). Fish histopathology as biomarker to evaluate water quality. Ecotoxicology and Environmental Contamination, 8(2), 09 - 15. https://doi.org/10.5132/eec.2013.02.002

Lins, J. A. P. N., Kirschnik, P. G., Queiroz, V. S., \& Cirio, S. M. (2010). Uso de peixes como biomarcadores para monitoramento ambiental aquático. Revista Acadêmica: Ciências Agrárias e Ambientais, 8(4), 469 - 484. http://dx.doi.org/10.7213/cienciaanimal.v8i4.11018

Mariano, W. S., Azevedo, S. B., Gomes, F. L., Lima, L. B., Moron, S. E., \& Tavares-Dias, M. (2019). Physiological parameters of Piaractus mesopotamicus (Osteichthyes: Characidae) exposed to a biopesticide based on Bacillus thuringiensis. Anais da Academia Brasileira de Ciências, 91(2). 10.1590/00013765201920180474

Martins, E. S., Aguiar, R. W. S., Martins, N. F., Melatti, V. M., Falcão, R., \& Gomes, A. C. M. M., Ribeiro, B. M., \& Monnerat, R. G. (2008). Recombinant Cry1Ia protein is highly toxic to cotton boll weevil (Nathonomus grandis Boheman) nad fall armyworm (Spodoptera frugiperda). Journal of Applied Microbiology, 104, 1363 - 1371. 10.1111/j.1365-2672.2007.03665x

Meher, S. M., Bodhankar, S. L., Arunkumar, Dhuley, J. N., Khodape, D. J., \& Naik, S. R. (2002). Toxicity studies of microbial insecticide Bacillus thuringiensis var. kenyae in rats, rabbits, and fish. Intenational Journal of Toxicology, 21, $99-105 . / 10.1080 / 10915810252866079$

Moraes, F. D., Venturini, F. P., Rossi, P. A., Avilez, I. M., Souza, N. E. S., \& Moraes, G. (2018). Assessment of biomarkers in the neotropical fish Brycon amazonicus exposed to cypermethrin-based insecticide. Ecotoxicology, 27, 188 - 197. https://link.springer.com/article/10.1007/s10646-017-1884-2

Mossa, A-T. H., Mohafrash, S. M. M., \& Chandrasekaran, N. (2018). Safety of natural insecticides: toxic effects on experimental animals. BioMed Research International. 10.1155/2018/4308054

Murussi, C. R., Menezes, C. C., Nunes, M. E. M., Araújo, M. C. S., Quadros, V. A., Rosemberg, D. B., \& Loro, V. L. (2015). Azadirachtin, a neem-derived biopesticide, impairs behavioral and hematological parameters in carp (Cyprinus carpio). Environmental Toxicology, $31(11), 1381-1388.10 .1002 /$ tox.22143

Oliveira, A. R., Castro, T. R., Capalbo, D. M. F., \& Delalibera Jr., I. (2007). Toxicological evaluation of genetically modified cotton (Bollgard®) and Dipel® WP on the non-target soil mite Scheloribates praeincisus (Acari: Oribatida). Experimental and Applied Acarology, 41, 191 - 201. 10.1007/s10493-007-9059-0

Oliveira-Filho, E. C. (2008). Avaliação da periculosidade ambiental de bioinseticidas como uma nova perspectiva para a ecotoxicologia no Brasil. Journal of the Brazilian Society of Ecotoxicology, 3(1), 1 - 7. 10.5132/JBSE.2008.01.001

Omoya, F. O., \& Akharaiyi, F. C. (2015). Physiological changes in Clarias gariepinus induced with Bacillus species used as biological agent in aquatic environment. Journal of Scientific Research \& Reports, 7(2), 117 - 128. 10.9734/JSRR/2015/8711

Pacheco, M., \& Santos, M. A. (2002). Biotransformation, genotoxic, and histopathological effects of environmental contaminants in European eel (Anguilla anguilla L.). Ecotoxicology and Environmental Safety, 53(3), 331 - 347. 10.1016/s0147-6513(02)00017-9

Paulino, M. G., Tavares, D., Terezan, A. P., Sakuragui, M. M., Posenti, E., Giani, A., Cestari, M. M., Fernandes, J, B., \& Fernandes, M. N (2020). Biotransformations, Antioxidant System Responses, and Histopathological Indexes in the Liver of Fish Exposed to Cyanobacterial Extract. Environmental Toxicology and Chemistry, v.39, n. 5, 1041-1051. 10.1002/etc.4696

Pino-Otín, M. R., Ballestero, D., Navarro, E., González-Coloma, A., Val, J., \& Mainar, A. M. (2019). Ecotoxicity of a novel biopesticide from Artemisia absinthium on non-target aquatic organisms. Chemosphere, 216, $131-146.10 .1016 /$ j.chemosphere.2018.09.071

Ranzani-Paiva, M. J. T., Lombardi, J. V., Maiorino, F. C., Gonçalves, A., \& Dias, D. C. (2014). Hematologia e histopatologia de tilápia-do-nilo exposta a concentrações sub-letais de selenito de sódio $\left(\mathrm{Na}_{2} \mathrm{SeO}_{3} \mathrm{Se}_{4}^{+}\right)$. Boletim do Instituto de Pesca, $40(1)$, $23 \quad-\quad 33$. https://www.pesca.agricultura.sp.gov.br/boletim/index.php/bip/article/view/1017

Sadauskas-Henrique, H., Sakuragui, M. M., Paulino, M. G., \& Fernandes, M. N. (2010). Using condition factor and blood variable biomarkers in fish to assess water quality. Environ Monit Assess, 181, 29-42 10.1007/s10661-010-1810-z

Santos, R. F. B., Dias, H. M., \& Fujimoto, R. Y. (2012). Acute toxicity and histopathology in ornamental fish amazon bluespotted corydora (Corydoras melanistius) exposed to formalin. Anais da Academia Brasileira de Ciências, 84(4). 10.1590/S0001-37652012000400014

Schweizer, M., Miksch, L., Köhler, H., \& Triebskorn, R. (2019). Does Bti (Bacillus thuringiensis var. israelensis) affect Rana temporaria tadpoles? Ecotoxicology and Environmental Safety, 181, 121 - 129. 10.1016/j.ecoenv.2019.05.080

Shaban, N. Z., Helmy, M. H., El-Kersh, M. A. R., \& Mahmoud, B. F. (2003). Effects of Bacillus thuringiensis toxin on hepatic lipid peroxidation and freeradical scavengers in rat given alpha-tocopherol or acetylsalicylate. Comparative Biochemistry and Physiology Part C, 135, 405 - 414. 10.1016/s15320456(03)00142-x

Sharma, N., \& Singhvi, R. (2017). Effects of Chemical Fertilizers and Pesticides on Human Health and Environment: A Review. International Journal of Agriculture, Environment and Biotechnology, 10(6), 675-679. 10.5958/2230-732X.2017.00083.3

Silva, A. G., \& Martinez, C. B. R. (2007). Morphological changes in the kidney of a fish living in an urban stream. Environmental Toxicology and Pharmacology, 23, 185 - 192. 10.1016/j.etap.2006.08.009

Silva, J. M., \& Santos, J. R. (2007). Toxicologia de agrotóxicos em ambientes aquáticos. Oecologia Brasiliensis, $11(4), 565$ - 573.

Udroiu, I. (2006). The micronucleus test in piscine erythrocytes. Aquatic Ecotoxicology, 79, 201 - 204. 10.1016/j.aquatox.2006.06.013

Venter, H. J., \& Bohn, T. (2016). Interactions between Bt crops and aquatic ecosystems: a review. Environmental Toxicology and Chemistry, 35(12), 2891 2902. 10.1002/etc. 3583 
Research, Society and Development, v. 10, n. 8, e2910816775, 2021

(CC BY 4.0) | ISSN 2525-3409| http://dx.doi.org/10.33448/rsd-v10i8.16775

Ventura, B. C., Angelis, D. F., \& Marin-Morales, M. A. (2008). Mutagenic and genotoxic effects of the Atrazine herbicide in Oreochromis niloticus (Perciformes, Cichlidae) detected by the micronuclei test and the comet assay. Pesticide Biochemistry and Physiology, $90,42-51$. 10.1016/j.pestbp.2007.07.009

Vieira, C. E. D., Costa, P. G., Cabrera, L. C., Primel, E. G., Fillmann, G., Bianchini, A., \& Martinez, C. B. R. (2017). A comparative approach using biomarkers in feral and caged Neotropical fish: Implications for biomonitoring freshwater ecosystems in agricultural areas. Science of the Total Environment, 586, $598-609.10 .1016 /$ j.scitotenv.2017.02.026

Villaverde, J. J., Sevilla-Morán, B., Sandín-España, P., López-Goti, C., \& Alonso-Prados, J. L. (2014). Biopesticides in the framework of the European Pesticide Regulation (EC) No. 1107/2009. Pest Management Science, 70, 2 - 5. 10.1002/ps.3663

Wolf, J. C., Baumgartner, W. A., Blazer, V. S., Camus, A. C., Engelhardt, J. A., Fournie, J. W., Frasca Jr., S., Groman, D. B., Kent, M. L., Khoo, L. H., Law, J. M., Lombardini, E. D., Ruehl-Fehlert, C., Segner, H. E., Smith, S. A., Spitsbergen, J. M., Weber, K., \& Wolfe, M. J. (2015). Nonlesions, misdiagnoses, missed diagnoses, and other interpretive challenges in fish histopathology studies: a guide for investigators, authors, reviewers, and readers. Toxicologic Pathology, 43(3), 297 - 325. 10. 1177/0192623314540229.

Wolf, J. C., \& Wolfe, M. J. (2005). A brief overview of nonneoplastic hepatic toxicity in fish. Toxicologic Pathology, $33(1), 75-85$. $10.1080 / 01926230590890187$ 